\title{
BMJ open TRIM59, a novel multiple cancer biomarker for immunohistochemical detection of tumorigenesis
}

\author{
Vida Khatamianfar, ${ }^{1}$ Fatma Valiyeva, ${ }^{1}$ Paul S Rennie, ${ }^{2}$ Wei-yang Lu, ${ }^{3}$ \\ Burton B Yang, ${ }^{4}$ Glenn S Bauman, ${ }^{5}$ Madeleine Moussa, ${ }^{6}$ Jim W Xuan ${ }^{1}$
}

To cite: Khatamianfar V, Valiyeva F, Rennie PS, et al. TRIM59, a novel multiple cancer biomarker for immunohistochemical detection of tumorigenesis. BMJ Open 2012;2:e001410. doi:10.1136/bmjopen-2012001410

- Prepublication history and additional material for this paper are available online. To view these files please visit the journal online (http://dx.doi.org/10.1136/ bmjopen-2012-001410).

Received 3 May 2012 Accepted 20 July 2012

This final article is available for use under the terms of the Creative Commons Attribution Non-Commercial 2.0 Licence; see http://bmjopen.bmj.com

For numbered affiliations see end of article

Correspondence to Jim W Xuan;

jim.xuan@lhsc.on.ca

\section{ABSTRACT}

Objectives and design: We identified a novel TRIM59 gene, as an early signal transducer in two (SV40Tag and Ras) oncogene pathways in murine prostate cancer (CaP) models. We explore its clinical applications as a multitumour marker detecting early tumorigenesis by immunohistochemistry (IHC).

Setting and participants: $88 \mathrm{CaP}$ patients were from a tissue microarray (TMA) of radical prostatectomy specimen, 42 patients from a 35 multiple tumour TMA, 75 patients with renal cell carcinoma (RCC) and 92 patients from eight different tumour groups (breast, lung, parotid, gastrointestinal, female genital tract, bladder, kidney and prostate cancer).

Results: TRIM59 upregulation specifically in tumour area was determined by IHC in 291 cases of 37 tumour types. To demonstrate that TRIM59 upregulation is 'tumour-specific', we characterised a significant correlation of TRIM59 IHC signals with tumorigenesis and progression, while in control and normal area, TRIM59 IHC signal was all negative or significantly low. TRIM59 protein upregulation in prostate and kidney cancers was detectable in both intensity and extent in early tumorigenesis of prostate intraepithelial neoplasia $(p<0.05)$ and grade 1 of RCC $(p<0.05)$, and stopped until high grades cancer. The results of the correlation in these two large cohorts of tumour types confirmed and repeated murine $\mathrm{CaP}$ model studies. Enhanced TRIM59 expression was identified in most of the 37 different tumours, while the highest intensities were in lung, breast, liver, skin, tongue and mouth (squamous cell cancer) and endometrial cancers. Multiple tumour upregulation was further confirmed by comparing relative scores of TRIM59 IHC signals in eight tumours with a larger patient population; and by a mouse wholemount embryo ( 14.5 days post conception) test on the origin of TRIM59 upregulation in epithelial cells.

Conclusions: TRIM59 may be used a novel multiple tumour marker for immunohistochemical detecting early tumorigenesis and could direct a novel strategy for molecular-targeted diagnosis and therapy of cancer.

\section{INTRODUCTION}

The TRIM (TRIpartite Motif) family is an evolutionarily conserved gene family implicated in a number of critical processes including

\section{ARSTICLE SUMMARY}

Article focus

- We identified a novel TRIM59 gene, as an early signal transducer in two (SV40Tag and Ras) oncogene pathways in murine prostate cancer (CaP) models.

- This is the first report on the clinical application of TRIM59 as a biomarker for multiple human cancers detecting early tumorigenesis by immunohistochemistry.

Key messages

- We first emphasised that the antibody utilised is TRIM59-specific in this work (also in the sister paper).

- Several TRIM59 antibodies obtained even commercially by immunogens of oligo-peptides may not be detecting the true TRIM59 protein.

- To demonstrate that TRIM59 upregulation is tumor tissue specific in multiple tumor tissues, we have presented sufficient controls, including negative staining in most of normal tissues.

- TRIM59 upregulation specifically in tumor area was determined by immunohistochemistry in 291 cases of 37 tumor types.

- In all, $88 \mathrm{CaP}$ patients were from a tissue microarray (TMA) of radical prostatectomy specimen, 42 patients from a 35 multiple tumor TMA, 75 patients with renal cell carcinoma (RCC) and 92 patients from 8 different tumor groups (breast, lung, parotid, gastrointestinal, female genital tract, bladder, kidney and prostate cancer).

- TRIM59 protein upregulation were detectable in both intensity and extent in early tumorigenesis of PIN (prostate intraepithelial neoplasia, $p<0.05)$ and grade 1 of RCC $(p<0.05)$, and stopped until high grades cancer.

- Enhanced TRIM59 expression was identified in most of the 37 different tumors, while the highest intensities were in lung, breast, liver, skin, tongue and mouth (squamous cell cancer) and endometrial cancers.

- Multiple tumor upregulation was further confirmed by comparing relative scores of TRIM59 IHC signals in eight tumors with a larger patient population; and by a mouse whole-mount embryo (14.5 days post conception) test on the origin of TRIM59 up regulation in epithelial cells. 


\section{STRENGTHS AND LIMITATIONS OF THIS STUDY}

- TRIM59 may be used a novel multiple tumor marker for immunohistochemical detecting early tumorigenesis and could direct a novel strategy for molecular-targeted diagnosis and therapy of cancer.

- The work was mostly on human tumor samples by immunohistochemistry.

immunity, ${ }^{1-3}$ antiviral, ${ }^{4-8}$ proliferation, ${ }^{6}{ }^{9}$ transcriptional regulation, ${ }^{6}{ }^{10}$ neuro-development, ${ }^{11}{ }^{12}$ cell differentiation $^{12}$ and cancer $^{13}$ (reviewed in refs ${ }^{1} 51^{14}{ }^{15}$ ). However, the function of most TRIM family members is poorly understood and was surmised only based on computational analysis from their RBCG (RING finger, $B$-box, Coiled-Coil) domains. The domain of RING (Really Interesting New Gene) is frequently involved in proteolysis acting as E3 ubiquitin ligases and the ubiquitin-proteasome system in the regulation of numerous cellular processes including cell cycle regulatory proteins, transcription factors and signal transducers. ${ }^{6}{ }^{15}$ Recent studies demonstrate that various TRIM-NHL proteins function as cofactor for the microRNA-induced silencing complex. ${ }^{12} 1416$ An ataxia-telangiectasia group $\mathrm{D}$ complementing gene (ATDC) was recently designated as TRIM29, which is elevated in most invasive pancreatic cancers in the Wnt/ $\beta$-catenin signalling pathway. ${ }^{13}$

In a previous report, ${ }^{17}$ we characterised the function of TRIM59, a novel TRIM family member, in SV40 Tag oncogene-directed genetically engineered mouse (GEM) prostate cancer (CaP) models. ${ }^{18-21}$ The TRIM59 gene was identified to be correlated with the SV40 Tag-initiated tumorigenesis. TRIM59 protein upregulation and hyperphosphorylation started in the prostate cytoplasm in early tumorigenesis from PIN (prostate intraepithelial neoplasia). ${ }^{17}$ As a signal pathway effector, the p-Ser/Thr phosphorylated TRIM59 proteins correlate with tumorigenesis, while p-Tyr-TRIM59 proteins correlate with advanced CaP. The function of TRIM59 was identified by shRNA knockdown in human CaP cells resulted in S-phase arrest and cell growth retardation. Although TRIM59 is an effector gene with the SV40Tag oncogene, the initial functional targets of TRIM59 function were actually on the Ras signal pathway as an early and rapid signal transmitter (for a review see ref. ${ }^{22}$ ). In a transgenic mouse test of TRIM59 upregulation specifically in the prostate, TRIM59 demonstrated full oncogenic activity in directing tumorigenesis and progression to highest-grade $\mathrm{CaP}^{17}{ }^{23}$ The signal pathway of TRIM59 may be possibly linked with two large oncogene routes: the Ras/Raf/MEK/ERK/PI3K/AKT and the SV40 Tag/ p53/pRB routes. ${ }^{17}$ In this study, we explored the clinical utility of the novel TRIM59 gene to serve as a biomarker for a panel of human cancers. To address the molecular biology basis, we combined results from both basic (animal model ${ }^{17-21}$ ) and human clinical studies.
MATERIALS AND METHODS

\section{Patient selection}

All patient samples were acquired as part of REB (Research Ethics Board) approved protocols at the Western University (UWO) and Vancouver Prostate Center, University of British Columbia (UBC). Table 1 shows a complete list of 291 patients with 37 different tumour types examined in this study.

\section{Prostate cancer tissue microarrays}

Eighty-eight CaP patients between 2006 and 2008 who had no treatment prior to radical prostatectomy were selected from the Vancouver General Hospital, UBC. Each patient block marked as containing benign tissue or cancer was sampled two times with a core diameter of $1 \mathrm{~mm}$ arrayed (176 cores) in a rectangular pattern with $0.7 \mathrm{~mm}$ between the centres of each core, creating a duplicate tissue microarray (TMA) layout using a manual tissue micro arrayer (Beecher Instruments, Silver Spring, Maryland, USA). The TMA paraffin blocks, were sectioned into $0.5 \mu \mathrm{m}$ sections and mounted on the positively charged slides.

\section{Automated image, acquisition and analysis on} immunohistochemical staining of CaP-TMA

Immunohistochemical staining was conducted by Ventana autostainer model Discover XT (Ventana Medical System, Tucson, Arizona, USA) with enzyme-labelled biotin streptavidin system and solvent-resistant DAB Map kit (Ventana Medical System). TMA was scanned by Bliss Digital imaging system using $\times 20$ objective (Bacus Laboratories Inc., Centre Valley, Pennsylvania, USA) and stored in the Prostate Centre Saver (http//bliss.prostatecentre.com). A value on a four-point scale assigned to each core.

\section{Multiple tumour TMA construction}

Tissue samples from 42 patients that encompassed 35 distinct tumour subtypes were selected from London Laboratory Service Group, and the tumour bank in the Department of Pathology (UWO). TMA slides were constructed with triplicate cores for each sample following standard procedure as described. ${ }^{24}$ Sections of $0.6 \mathrm{~mm}$ were prepared from TMA block and re-stained by H\&E for each case to confirm the diagnosis.

\section{Histopathological analysis}

All cases from 37 tumour types were graded according to standardized histopathology grading systems ${ }^{25}$ by MM (pathologist) and VK (MD fellow).

\section{Immunohistochemistry and results evaluation}

Standard ABC (Avidin Biotin Complex) protocol was performed as previously reported. ${ }^{21}{ }^{26-29}$ Two kinds of blocking reagents were used: Power Block (Universal Blocking Reagent; BioGenex, San Ramon, California, USA) and Avidin-biotin blocking reagent kit (Vector Labs, Burlingame, California, USA). All B-Raf antibodies were from GenScript (Piscataway, New Jersey, USA): B-Raf 
Table 1 Patient list selected in this study

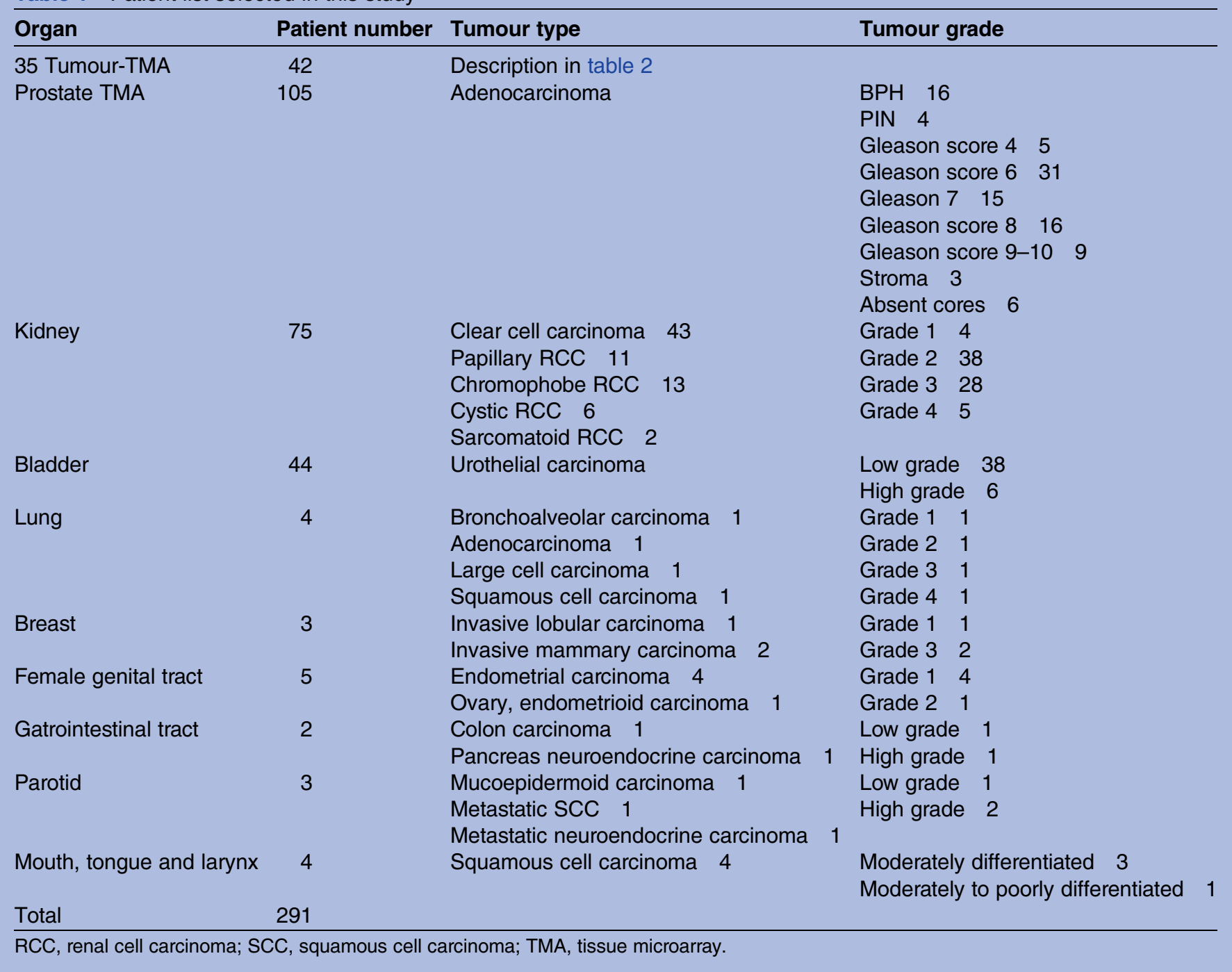

antibody (monoclonal antibody, used 1:500 dilution), Raf 1 (polyconal, 1:50) and Raf -1 (polyclonal, Phospho-Ser $\left.{ }^{259} 1: 50\right)$.

TRIM59 IHC staining signals were assessed by intensity for cytoplasmic staining and extent to assess the percentage of nuclear staining as previously reported. ${ }^{28}{ }^{29}$ Since in some tumours TRIM59 showed only cytoplasmic staining, for the purpose of comparing in different tumours, we used a combined relative score system based on both intensity and extent as follows: score 0: 0/0 (intensity/ extent); score 1: weak cytoplasmic staining and/or $\leq 25 \%$ nuclear staining; score 2: moderate cytoplasmic staining and/or $\leq 50 \%$ nuclear staining; score 3: strong cytoplasmic staining and/or $\geq 50 \%$ nuclear staining. All relative scores were accessed by two researchers independently MM (pathologist) and VK (MD fellow).

\section{Construction and characterisation of TRIM59 antibody}

Online supplementary figure S1 provided details, which combined online supplementary materials of, ${ }^{17}$ and more informations. Online supplementary figure S1A showed that the antibody TRIM59\#72 used for mouse and human studies was prepared from C-terminal 136-amino acid peptide (as recombinant GST-fusion protein, see online supplementary figure S1B) of wholeprotein 403aa which is TRIM59 specific, while antibody TRIM59\#71 was from mouse TRIM59 N-terminal sequence (163aa) containing mostly the common RBCC function domains of more than 70 members the TRIM family. ${ }^{17}$ As shown in online supplementary figure S1C, western blots on both mouse and human cell lysates, \#72 recognised only one band, while \#71 showed multiple bands. We also demonstrated that N-terminal sequencespecific antibody TRIM59\#71 can recognise purified proteins from C-terminal sequence-specific, TRIM59\#72 Ab affinity column (the same $53 \mathrm{kDa}$ protein, see online supplementary figure S1D). Since these two immunogenic sequences are not overlapped, we thus verified that they are detecting the same protein. We demonstrated that TRIM59\#72 can be used for human tissues for immunohistochemistry (IHC) and western blots (see online supplementary figure S1E). 
Table 2 Immunohistochemistry (IHC) analysis of TRIM59 as multiple marker in 35 tumour tissue microarray

\begin{tabular}{|c|c|c|c|c|c|c|}
\hline \multirow[b]{2}{*}{ Tumour type } & \multirow[b]{2}{*}{$\begin{array}{l}\text { Patient } \\
\text { number }\end{array}$} & \multirow[b]{2}{*}{$\begin{array}{l}\text { Core } \\
\text { number }\end{array}$} & \multirow[b]{2}{*}{$\begin{array}{l}\text { Pathological } \\
\text { grade }\end{array}$} & \multirow[b]{2}{*}{ Cell type } & \multicolumn{2}{|l|}{ IHC staining } \\
\hline & & & & & $\begin{array}{l}\text { Cytoplasm } \\
\text { staining (intensity) }\end{array}$ & $\begin{array}{l}\text { Nuclear } \\
\text { staining (extent) }\end{array}$ \\
\hline \multirow[t]{2}{*}{ Renal clear cell carcinoma, } & 2 & 6 & 2 & Epithelial & moderate & - \\
\hline & & & 3 & & moderate & - \\
\hline Adrenal gland cortical carcinoma & 1 & 3 & $\mathrm{~N} / \mathrm{A}$ & Epithelial & Moderate-strong & - \\
\hline \multirow{2}{*}{$\begin{array}{l}\text { Squamous cell carcinoma } \\
\text { (SCC), skin }\end{array}$} & 2 & 6 & WD & Epithelial & Strong & - \\
\hline & & & MD & & Moderate & - \\
\hline Basal cell carcinoma, skin & 2 & 6 & $\mathrm{~N} / \mathrm{A}$ & Epithelial & $\begin{array}{l}\text { Moderate } \\
\text { Moderate-strong }\end{array}$ & - \\
\hline Melanoma & 1 & 3 & $\mathrm{~N} / \mathrm{A}$ & Epithelial & Weak & $50 \%+$ \\
\hline \multirow[t]{2}{*}{ Endometroid adenocarcinoma } & 2 & 6 & 2 & Epithelial & Moderate-strong & - \\
\hline & & & 1 & & Moderate-strong & - \\
\hline Leiomyosarcoma & 1 & 3 & $\mathrm{~N} / \mathrm{A}$ & Mesenchymal & Weak & - \\
\hline $\begin{array}{l}\text { Omentum serous } \\
\text { adenocarcinoma, }\end{array}$ & 1 & 3 & WD & Epithelial & Weak-moderate & - \\
\hline Ovary serous adenocarcinoma & 1 & 3 & $\mathrm{~N} / \mathrm{A}$ & Epithelial & Weak-moderate & - \\
\hline Ovary clear cell carcinoma & 1 & 3 & PD & Epithelial & Moderate-strong & $30 \%+$ \\
\hline Cervix adenocarcinoma & 1 & 3 & WD-MD & Epithelial & Moderate-strong & - \\
\hline Colon adenocarcinoma & 1 & 3 & Low grade & Epithelial & Weak & - \\
\hline Breast ductal adenocarcinoma & 1 & 3 & $2 / 3$ & Epithelial & Moderate-strong & $50 \%+$ \\
\hline \multirow[t]{2}{*}{ Bladder urothelial carcinoma } & 2 & 6 & Low grade & Epithelial & Weak & - \\
\hline & & & 2 (low grade) & Epithelial & Moderate & - \\
\hline Stomach GIST & 1 & 3 & & Epithelial & Weak-moderate & - \\
\hline Esophagus adenocarcinoma & 1 & 3 & MD & Epithelial & 0-Weak & - \\
\hline Thyroid, papillary carcinoma & 1 & 3 & $\mathrm{~N} / \mathrm{A}$ & Epithelial & Weak & - \\
\hline Thyroid, medullary carcinoma & 1 & 3 & $\mathrm{~N} / \mathrm{A}$ & Epithelial & Moderate & - \\
\hline \multirow[t]{2}{*}{ Pancreas adenocarcinoma } & 2 & 6 & 2 & Epithelial & Weak-moderate & - \\
\hline & & & 2 & & Moderate-strong & - \\
\hline Pancreas endocrine tumour & 1 & 3 & $\mathrm{~N} / \mathrm{A}$ & Epithelial & Strong & - \\
\hline Lung SCC & 1 & 3 & PD & Epithelial & Strong & - \\
\hline Lung mesothelioma & 1 & 3 & MD-PD & & Strong & $20 \%+$ \\
\hline Lung adenocarcinoma & 1 & 3 & MD & & Moderate & $50 \%+$ \\
\hline Lung bronchoalveolar carcinoma & 1 & 3 & WD & & Moderate-strong & $50 \%+$ \\
\hline Lung mesothelioma, biphasic & 1 & 3 & MD-PD & & Strong & $20 \%+$ \\
\hline $\begin{array}{l}\text { Liver hepatocellular carcinoma } \\
\text { (HCCa) }\end{array}$ & 1 & 3 & $2 / 4$ & Epithelial & Strong & - \\
\hline Liver metastatic carcinoid & 1 & 3 & $\mathrm{~N} / \mathrm{A}$ & Epithelial & Strong & - \\
\hline $\begin{array}{l}\text { Small bowel marginal zone } \\
\text { lymphoma }\end{array}$ & 1 & 3 & $\mathrm{~N} / \mathrm{A}$ & Lymphocyte & 0-Weak & - \\
\hline Lymph node, follicular lymphoma & 1 & 3 & $1 / 3$ & Lymphocyte & 0-Weak & - \\
\hline $\begin{array}{l}\text { Lymph node, metastatic } \\
\text { carcinoid }\end{array}$ & 1 & 3 & Low grade & Epithelial & Strong & - \\
\hline Spleen, Hodgkin's lymphoma & 1 & 3 & $\mathrm{~N} / \mathrm{A}$ & Lymphocyte & Weak & - \\
\hline Stomach, malt lymphoma & 1 & 3 & Low grade & Lymphocyte & 0-Weak & - \\
\hline Thymus invasive thymoma & 1 & 3 & N/A & Epithelial & 0-Wweak & - \\
\hline Appendix, goblet cell carcinoid & 1 & 3 & $\mathrm{~N} / \mathrm{A}$ & Epithelial & 0 & - \\
\hline
\end{tabular}

Confocal microscope imaging of immunostaining of mouse embryos 14.5 days post conception for TRIM59

The mouse embryos were prepared according to our previous reports. ${ }^{19} 20$ The anti-rabbit secondary antibody was conjugated to Cy3 (1:200, Jackson Immuno Research, West Grove, Pennsylvania, USA). Sections were doublestained with pan-cytokeratin antibody with FICT (Sigma, St. Louis, Missouri, USA), and the stained mouse embryo sections were visualised using a Carl Zeiss (Oberkochen, Germany) confocal microscope by the LSM Image program.

\section{Statistical analysis}

Student's t tests and one-way analysis of variance were used by programs of Microsoft Excel 2007 or SPSS 10 to 
analyse the data with $\mathrm{p}<0.05$ considered to be statistically significant.

\section{RESULTS AND DISCUSSION}

TRIM59 upregulation in human prostate cancer TMA: correlation with tumorigenesis and tumor progression by TRIM59 intensity until high grade CaP

We designated TRIM59 as one of the 'tumorigenesis-associated' genes correlated with SV40 Tag oncogenesis in mouse prostate cancer (GEM-CaP) models. ${ }^{17}$ SV40 Tag is essentially only required for the initiation of tumorigenesis, that is, the 'hit-and-run' effect, in GEM-CaP, but not for the tumour progression and metastasis directly. The 'tumorigenesis-associated' effect is due to the initial binding of the Tag oncogene with retinoblastoma ( $p R B)$, p53 proteins and several transcriptional coactivators. Once this process is initiated, the signal transduction will continue on, even without the initiation effectors.

In GEM-CaP models, the TRIM59 protein upregulation correlated with tumorigenesis and progression, and downregulated in the high-grade $\mathrm{CaP}$ by $\mathrm{IHC}^{.17}$ We assume this 'tumorigenesis-associated' effect of TRIM59 may apply to human cancer studies.

We first characterised that TRIM59 antibody (\#72) can cross-react with and recognise specifically human TRIM59 counterpart (for details see online supplementary figure S1).
We tested by IHC in a TMA of CaP patients $(n=88,176$ cores). TRIM59 IHC signals detected in an automated digital image system were identified mostly in cytoplasm of luminal cells (figure 1A), which is different from rapid tumour progression mouse $\mathrm{CaP}$ models. The intensity $($ score $=2)$ in PIN $(n=4$, figure 1A) was higher than in non-tumour area (normal and benign prostatic hyperplasia). Moderate-to-strong expression was observed in Gleason score $6(3+3, n=25), 7(4+3, n=15)$ and $8(4+4$, $\mathrm{n}=14$ ). In high-grade CaP (score $4+5, \mathrm{n}=8$, figure $1 \mathrm{~A}$ ), TRIM59-IHC signals were lower. As shown in a graph of figure 1B, TRIM59 protein IHC signals correlated significantly $(\mathrm{p}=0.014)$ with tumorigenesis and progression from PIN to WDCaP (Gleason grade 1-2, scores 2-4) and MDCaP (Gleason grade 2-4, scores 4-8) (graph figure 1B), and decreased in high-grade $\mathrm{CaP}$ (Gleason score 9-10) with $\mathrm{p}=0.018$, which is similar to GEMs. ${ }^{17}$

\section{TRIM59 upregulation in human renal cell carcinoma} patients: correlation of tumorigenesis and tumour progression with TRIM59 intensity until high-grade RCC

Next, we demonstrated that TRIM59 is not androgenresponsive and likely not prostate tissue-specific, indicating that the tumorigenesis role of TRIM59 may be applied to all or most of human cancers. Online supplementary figure S2 showed this result in detail of IHC

\section{A. Prostate cancer TMA: IHC- TRIM59}
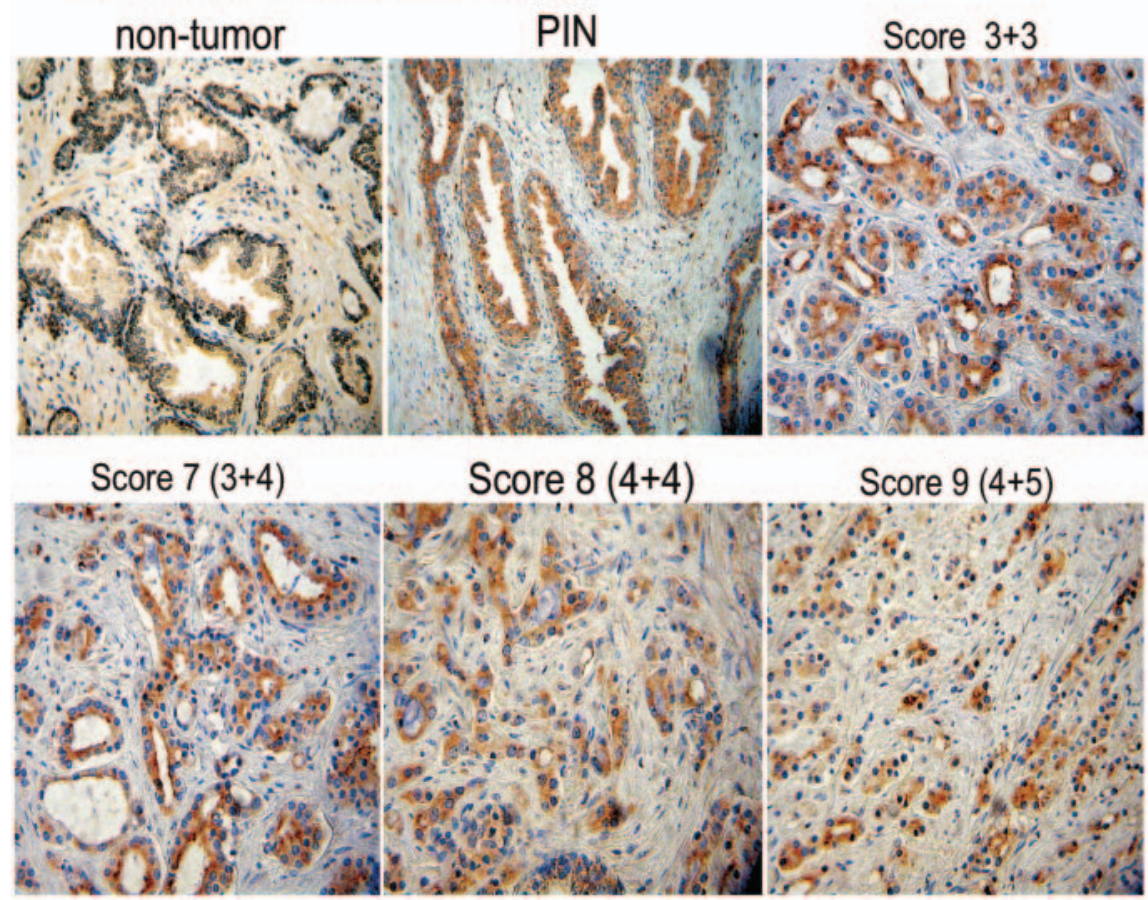

Score $3+3$
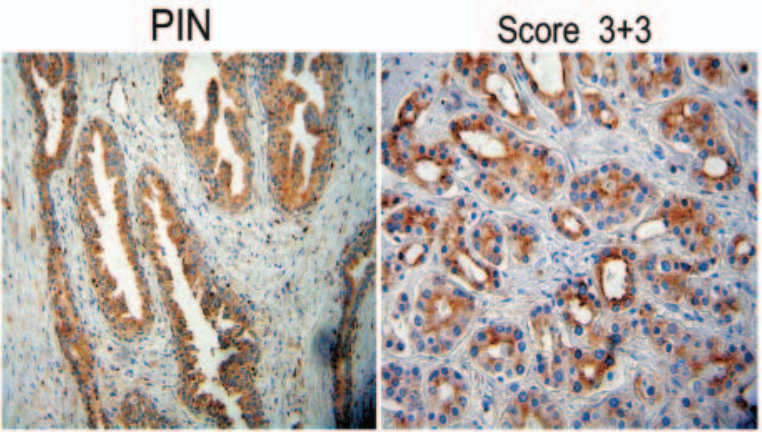

B. Correlation of TRIM59 IHC signals with Gleason scores in CaP-TMA

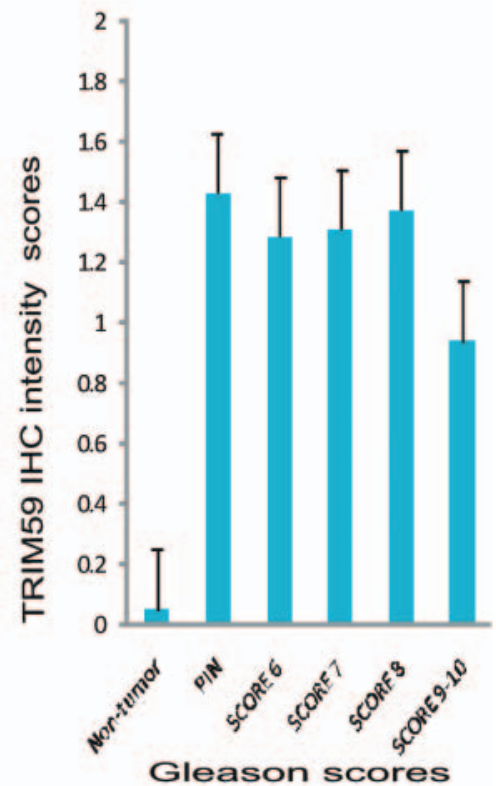

Figure 1 Correlation of TRIM59 immunhistochemistry (IHC) staining in prostate cancer cases in tissue microarray (TMA) assessed by the Gleason grading system. All panels in $(A)$ were shown haematoxylin staining, $\times 20$. Non-tumour: weak or negative, prostate intraepithelial neoplasia (PIN): TRIM59 is located in the cytoplasm of the luminal cells (intensity =2). Gleason score $3+3$, TRIM59 is located in the cytoplasm of tumour cells (intensity $=2$ ). Gleason score $3+4$, TRIM59 is located in the cytoplasm of the tumour cells (intensity $=2$ ). Gleason score $4+4$, TRIM59 is located in the cytoplasm of the tumour cells (intensity =2). Gleason score 4+5, TRIM59 is located in the cytoplasm of the tumour cells (intensity<1). Graph B: correlation of TRIM59 protein levels by intensity with Gleason score in prostate cancer TMA analysis. Error bars show mean $( \pm \mathrm{SE})$. 
test by TRIM59 antibody\#72 on a TMA of the LNCaP human $\mathrm{CaP}$ xenografts in nude/severe combined immuno deficiency castrated mice.

We extended results from prostate cancer clinical samples to kidney cancer. We started 75 renal cell carcinoma (RCC) patients including all 5 different types of RCC tumours: 43 clear cell carcinoma (representative IHC figures shown in figure 2A-D), 11 papillary RCC (figure 2E,F), 13 chromophobe RCC (figure 2G-I), 2 sarcomatoid RCC (figure 2J), and 6 cystic RCC (figure 2K,L). RCC cases analysed with Fuhrman grade $1-4$ were $4,38,28$ and 5, respectively. TRIM59-IHC staining in normal area including proximal tubules was negative (figure 2, last row). Background staining was eliminated by testing antibody dilutions $(1: 100$,
200, 500, 1200 till 5000) while tumour-specific signals of TRIM59 proteins were noticeable. Endogenous biotin signals were blocked and excluded by additional block reagents (avidin-biotin blocking reagent kit).

TRIM59 IHC staining in tumour areas in RCC (figure 2) was different from cases of CaP-TMA (mainly cytoplasmic). TRIM59 IHC staining was found in both cytoplasm and nucleus in all RCG grades and types (figure 2). We assessed TRIM59-IHC by visual scoring of both intensity (cytoplasmic staining) and extent (\% nucleus staining) microscopically. Correlation of TRIM59 IHC signals by scoring the intensity in cytoplasm with grades of all five types of RCC is shown in figure 2M. TRIM59 IHC signals were increased with tumour progression from grade 1-3

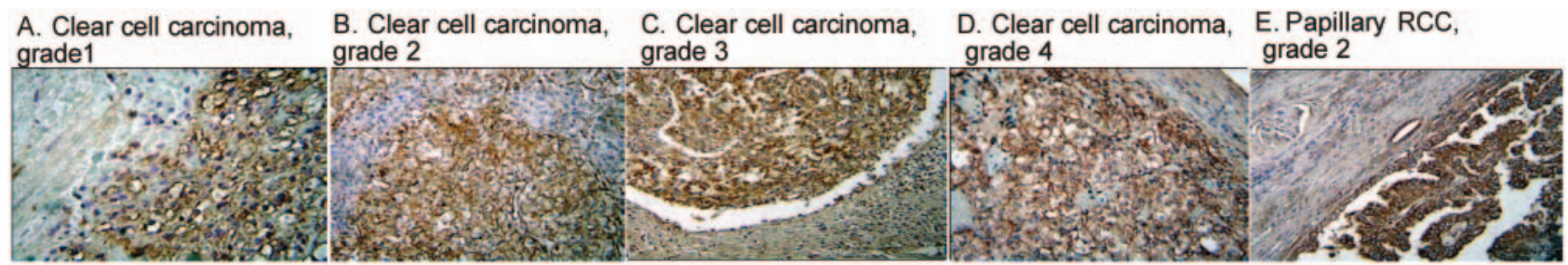

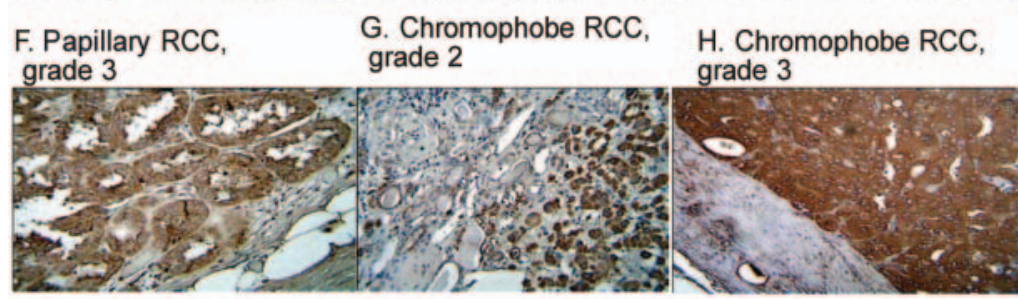
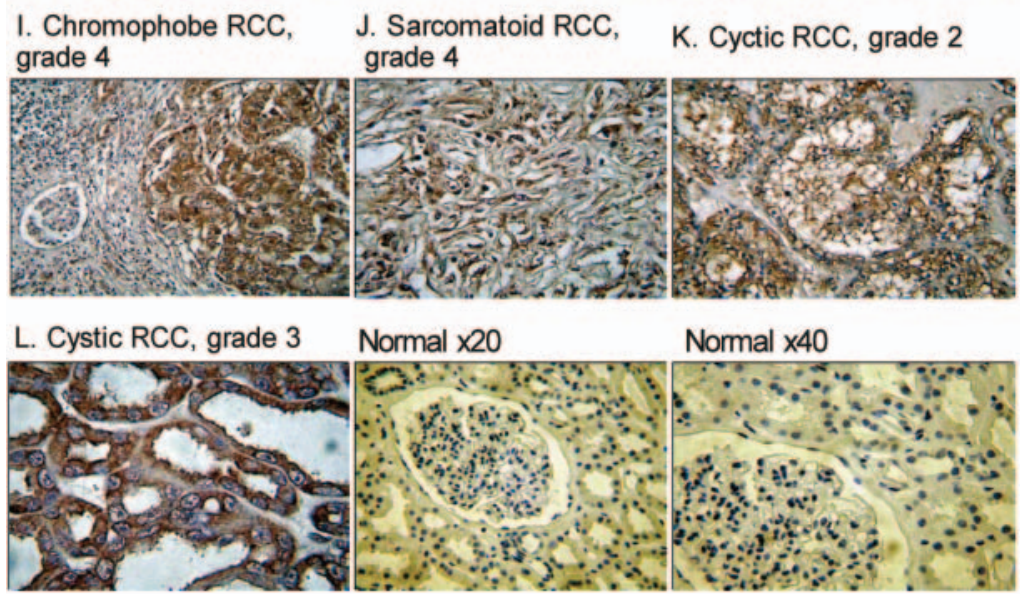

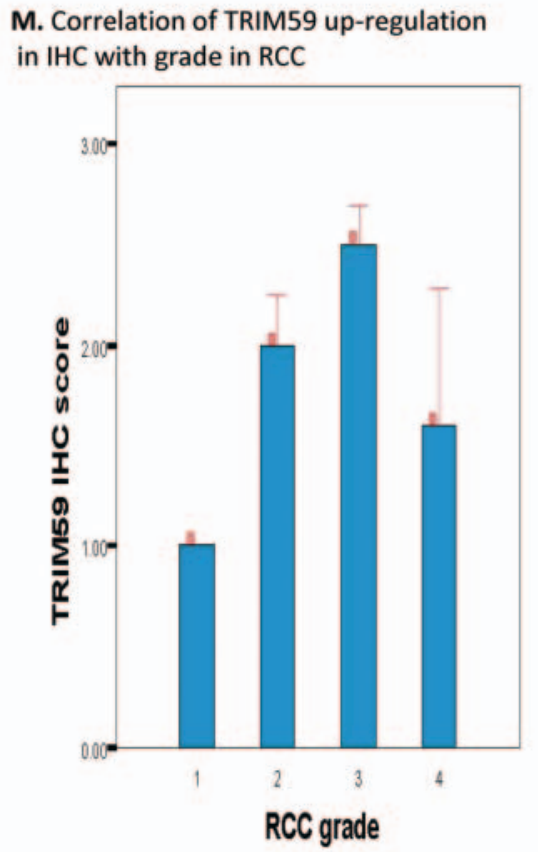

Figure 2 Immunohistochemistry analysis of TRIM59 expression in kidney cancer (RCC, renal cell carcinoma) cases: correlation with tumour grade by intensity detecting early tumorigenesis. Five types of RCC with different grades were shown clear cell carcinoma (A-D), papillary RCC (E and F), chromophobe RCC (G-I), sarcomatoid RCC (J) and cystic RCC (K and L). (A) Clear cell carcinoma, grade1, weak cytoplasmic staining in tumour cells, $\geq 50 \%$ nuclear staining, cytoplasmic staining in tumour cells $(\times 40)$. (B) Clear cell carcinoma, grade 2, moderate cytoplasmic staining in tumour cells, no nuclear staining $(\times 40)$. (C) Clear cell carcinoma, grade 3 , strong cytoplasmic staining in tumour cells, no nuclear staining $(\times 40)$. (D) Clear cell carcinoma, grade 4 , moderate cytoplasmic staining and nuclear (\%) staining $(\times 40)$. (E) Papillary RCC, grade 2, strong cytoplasmic staining in tumour cells, no nuclear staining $(\times 40)$. (F) Papillary RCC, grade 3 , strong cytoplasmic staining in tumour cells, no nuclear staining, normal cortex tissue is visible adjacent to tumour area $(\times 40)$. (G) Chromophobe RCC, grade 2, strong cytoplasmic staining in tumour cells, no nuclear staining. $(\times 40)$. $(\mathrm{H})$ Chromophobe RCC, grade 3 , strong cytoplasmic staining, no nuclear staining, $(\times 40)$. (I) Chromophobe RCC, grade 4, moderate cytoplasm staining and nuclear staining (x40). (J) Sarcomatoid RCC, grade 4, weak cytoplasmic staining and nuclear staining $(\times 40)$. (K) Cystic RCC, grade 2, moderate cytoplasmic staining, no nuclear staining ( $\times 40)$. (L)Cystic RCC, grade 3, strong cytoplasmic staining, no nuclear staining ( $\times 40)$. Last two panels: normal kidney tissues $(\times 20, \times 40)$. (M) Graph: correlation of TRIM59 protein levels by relative scores (both intensity and extent) with grade in RCC according to the Fuhrman nuclear grading system. Error bars show mean $( \pm \mathrm{SE})$. 
$(\mathrm{p}<0.05)$. All grade 1 tumours $(n=4)$ stained with weak TRIM59 IHC signals in cytoplasm, but with high extent of nuclear staining; while all grade 2 and 3 tumours $(n=66)$ showed moderate-to-strong cytoplasmic staining intensity of TRIM59. All grade 4 tumours $(n=5)$ showed weak-tomoderate intensity in cytoplasm of TRIM59 staining. No correlation between TRIM59 IHC staining in nucleus and tumour grade was found, although low-grade RCC showed higher nuclear staining.

Therefore, by systematic IHC studies in CaP ( 88 patients, figure 1) and kidney cancer (75 patients, figure 2), we almost exactly repeated results from our mouse model studies on TRIM59. ${ }^{17}$ We confirmed TRIM59 as an IHC marker able to detect low-grade tumour in early tumorigenesis. We also demonstrated the correlation of tumorigenesis and tumour progression with TRIM59 upregulation until high-grade tumour.

\section{TMA analysis of TRIM59 protein expression demonstrates that TRIM59 is a multiple tumour marker}

In the basic research previously using animal GEM-CaP models ${ }^{17}$ we have disclosed that TRIM59 upregulation is involved in two oncogene families and two signal pathways of SV40Tag/pRB/p53 and Ras/Raf/MEK/ERK. TRIM59 may function as an early signal transducer in Ras signal pathway with bridging genes in two oncogene pathways. ${ }^{17}$

While it was rarely reported that SV40 Tag oncogene induced human cancer, Ras mutations are among the most frequent alterations in human cancers (for a review see ref. ${ }^{22}$ ). We assume that TRIM59 as an early Ras signal pathway effector may possibly act as a multiple tumour marker.

We therefore further extended TRIM59 IHC studies to 35 multiple cancer TMA sections (42 tumours, 126 cores, table 1$)$. We tested different dilutions $(1 / 300,1 / 600$, $1 / 1200$ and $1 / 5000$ ) of TRIM59 antibody (see online supplementary figure S3). To further confirm the specificity and reliability of TRIM59 antibody in IHC staining, we compared IHC staining in 35 different tumour-TMA sections with positive (TRIM59 antibody at 1:1200 and 1:5000 dilutions) and negative controls (no antibody added, for details see online supplementary figure S4). As summarised in table 2, TRIM59 expression was significant and tissue-specifically upregulated in most of these 35 tumours. When comparing the relative scores (both intensity and extent) in different tumours, the highest staining was observed in breast, lung, liver, skin (squamous cell carcinoma) and endometrial cancers.

\section{Further confirmation of TRIM59 as a tumour marker in patients with eight different tumours}

Since the 35 tumour-TMA contained only limited cases in each tumour type, we selected more cases $(n=92)$ of eight different tumour types with different tumour grades, which all showed upregulated expression of TRIM59. IHC staining of TRIM59 in eight tumours are shown: lung $(\mathrm{n}=4$, figure $3 \mathrm{~A}-\mathrm{C})$, breast $(\mathrm{n}=3$, figure $3 \mathrm{D}, \mathrm{E})$, gastrointestinal $(\mathrm{n}=2$, figure $3 \mathrm{~F}, \mathrm{G})$, female genital tract $(\mathrm{n}=5$, figure $3 \mathrm{H}-\mathrm{J})$, bladder $(\mathrm{n}=44$, figure $3 \mathrm{~K})$, prostate $(\mathrm{n}=27$ from UWO, figure 3L), head and neck mucosal tumour (squamous cell carcinoma, SCC of mouth, tongue and larynx, $\mathrm{n}=4$, figure $3 \mathrm{M}-\mathrm{O}$ ) and parotid gland $(\mathrm{n}=3$, figure $3 \mathrm{P}, \mathrm{Q})$. Normal areas in lung, breast, colon, endometrial, bladder, larynx and parotid tissues showed very weak or completely negative staining (figure 3 ).

Since some tumours (eg, prostate) showed mostly cytoplasmic and no nuclear TRIM59-IHC staining, as a comparative study, we assessed their relative scores (combine both intensity and extent scores, see Materials and methods). More tumours from kidney (RCC, $n=75$ ) and prostate cancer $(\mathrm{n}=27)$ were included as references and all were assessed by relative scores simultaneously, since we already analysed a large cohort of these patients. Figure 3R shows the comparison of the mean of IHC-TRIM59 relative scores. The highest relative scores were found in SCC of the parotid, mouth, larynx and tongue, followed by lung, breast and female genital tract cancers.

The comparison of relative scores on low and high grades separately was done (data not shown). Cases of grade 1 lung cancer (bronchoalveolar, adenocarcinoma, squamous cell carcinoma (SCC) and large cell carcinoma) and breast cancer (invasive lobular and invasive mammary carcinoma) all showed the strongest staining as compared with other tumours. In endometrial cancer, the TRIM59 relative scores were moderate in grade 1 and moderate to strong in grade 2. The three tumours of SCC from mouth, tongue and larynx with different grades (figure $3 \mathrm{M}-\mathrm{O}$ ) also showed high relative scores (both intensity and extent).

As a comparative study (figure $3 \mathrm{R}$, table 2), we tested 44 bladder cancer cases with 38 low-grade and 6 highgrade tumours. The mean value of relative scores was 1.6, that is, weak to moderate in bladder cancer cases. In 27 prostate cancer cases (from UWO only) tested, the relative scores of TRIM59 (cytoplasmic staining only) from PIN through Gleason scores of 10 were actually relatively weak (figure $3 \mathrm{R}$ ), although in Gleason score 4, 6 and 8 were weak to moderate separately.

So far, we identified that TRIM59 upregulation is 'tumour-specific'. First, we demonstrated the correlation of TRIM59-enhanced IHC signals with tumorigenesis and progression, which were statistically significant in this report with 291 cases and 37 tumour type analyses. Second, although TRIM59 is a normal gene involved in CDC (cell cycle division) regulation from G1 to S-phase and involved in DNA S-phase and cell growth, ${ }^{17}$ we demonstrated that in normal or non-tumour areas in all tested 37 different kinds of cancers, TRIM59 IHC staining signals were mostly negative or very low (figures 1-3). By moderating antibody dilutions and testing various blocking reagents (see online supplementary figure S4), we demonstrated that TRIM59 induces tumorigenesis/oncogenesis only when it is abnormally upregulated. 


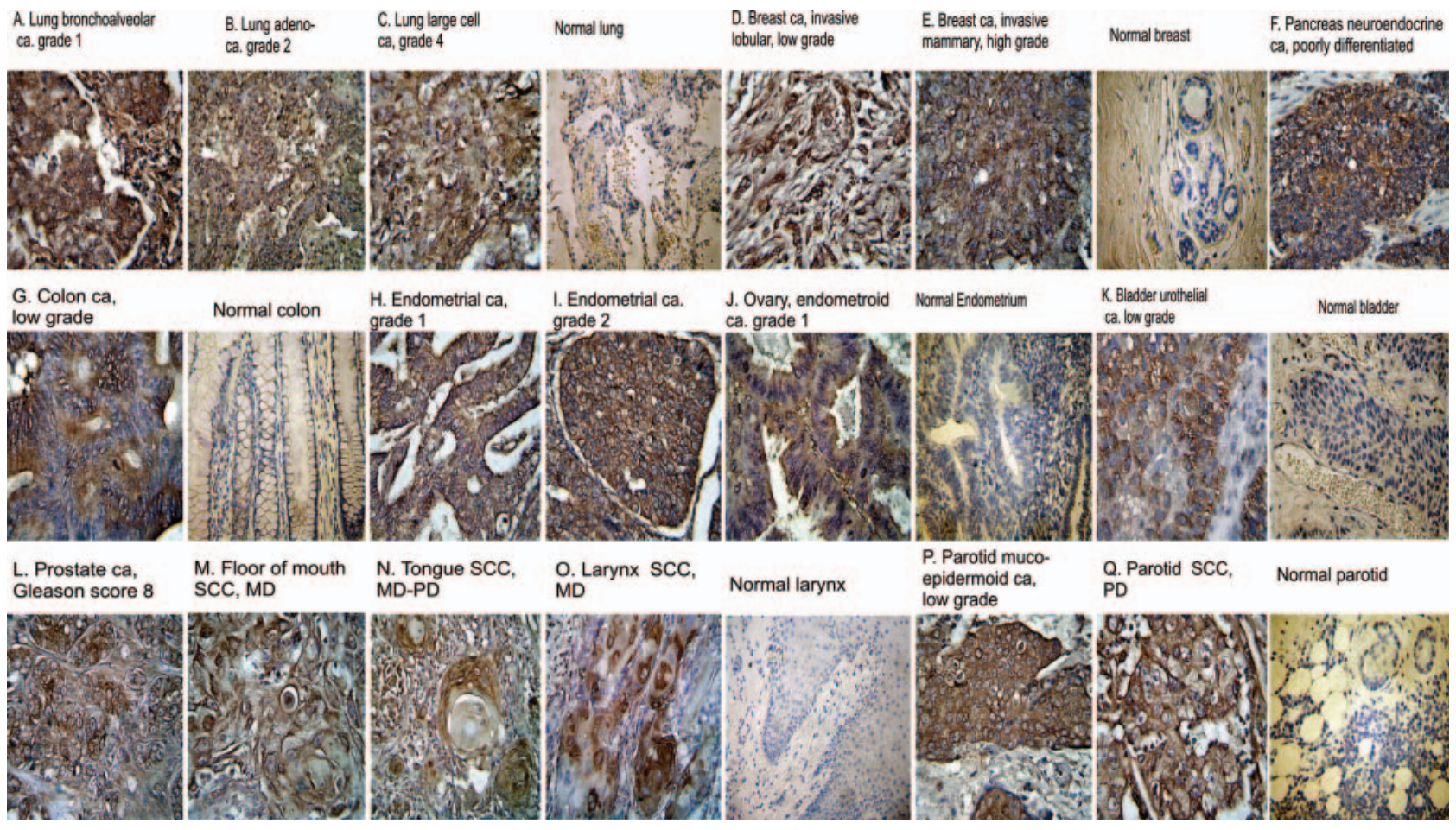

Figure 3 Comparison of TRIM59 expression as a multiple-cancer marker in eight types of tumours in breast, lung, parotid, gastrointestinal, female genital tract, bladder, head and neck mucosal tumour and prostate cancer. Negative TRIM59 staining in normal tissues was shown for each tumour, respectively (×20). (A) Lung bronchoalveolar carcinoma, grade 1, strong cytoplasmic and nuclear staining of tumour cells, $(\times 40)$. (B) Lung adenocarcinoma, grade 2, moderate cytoplasmic staining of tumour cells, no nuclear staining $(\times 40)$. (C) Lung large cell carcinoma, grade 4, moderate cytoplasmic and nuclear staining of tumour cells $(\times 40)$.

(D) Breast cancer, invasive lobular, low-grade, moderate to strong cytoplasmic and nuclear staining of tumour cells $(\times 40)$.

(E) Breast cancer, invasive mammary (no specific type), high-grade, moderate cytoplasmic staining of tumour cells, no nuclear staining $(\times 40)$. (F) Pancreas neuroendocrine carcinoma, poorly differentiated, strong cytoplasmic staining, no nuclear staining of tumour cells $(\times 40)$. (G) Colon carcinoma, low-grade, weak-to-moderate cytoplasmic staining of tumour cells, no nuclear staining $(\times 40)$. (H) Endometrial carcinoma, grade1, weak-to-moderate cytoplasmic staining of tumour cells, no nuclear staining $(\times 40)$. (I) Endometrial carcinoma, grade 2, moderate-to-strong cytoplasmic staining, no nuclear staining $(\times 40)$. (J) Ovary, endometrioid carcinoma, grade 1, weak cytoplasmic staining, no nuclear staining $(\times 40)$. $(K)$ Bladder urothelial carcinoma, low-grade, moderate cytoplasmic staining, no nuclear staining $(\times 40)$. (L) Prostate adenocarcinoma, Gleason score 8 , moderate cytoplasmic staining, no nuclear staining $(40 \times)$. (M) Floor of mouth, SCC moderately differentiated, strong cytoplasmic staining, no nuclear staining (40x). (N) Tongue cancer, SCC moderately to poorly differentiated, strong cytoplasmic staining, no nuclear staining (40x). (O) Larynx, SCC moderately differentiated, strong cytoplasmic staining, no nuclear staining (40x). (P) Parotid, mucoepidermoid carcinoma, low-grade, strong cytoplasmic staining, no nuclear staining $(\times 40)$. (Q) Parotid, metastatic SCC, poorly differentiated, moderate-to-strong cytoplasmic staining, no nuclear staining $(\times 40)$. $(R)$ Graphic comparison of relative scores (both intensity and extent) of TRIM59 IHC signals in eight different tumour types. fGT, female genital tract. Error bars show means $( \pm S E)$ of the relative scores in all grades analysed and compared.

In most of human cancers tested in this clinical IHC study, strong TRIM59 expression in tumour were identified in epithelial cancers: lung, breast, skin, which all associated with epithelium originations (very rare from mesenchymal tumours, for details see table 2).

Furthermore, we also confirmed that TRIM59 expression involved in multiple tissue expression even in embryo development. We carried out IHC of mouse embryo sections by double-staining TRIM59. Cytokeratin (keratin), a family of proteins that are primarily found in epithelial cells was used as reference. Online supplementary figure S5 illustrated confocal microscope images of IHC staining of TRIM59 in different organs/tissues of mouse embryo (14.5 days postconception). TRIM59 was highly expressed in cytokeratin-expressing cells in the lung (first row), skin (second row), and kidney (not shown) of mouse embryos. TRIM59 staining in mouse embryos revealed the same pattern of the epithelium origin as in human tumours, which the TRIM59 gene were found highly upregulated in those tumour types as well (see table 2).

Given our previous experiments suggesting TRIM59 functions in the Ras pathway, we tested if TRIM59 upregulation was correlated with the BRAF, an early signal effector Ras signal pathway (for a review see ref. ${ }^{22}$ ). We selected 24 RCG patients, which previously were confirmed with upregulation of TRIM59 expression. Three antibodies were used: B-Raf Antibody (mAb) and Raf-l Antibody (Ab-259) testing the total Raf protein and 


\section{R. Comparison of relative scores of TRIM59 IHC staining in eight different tumor types}

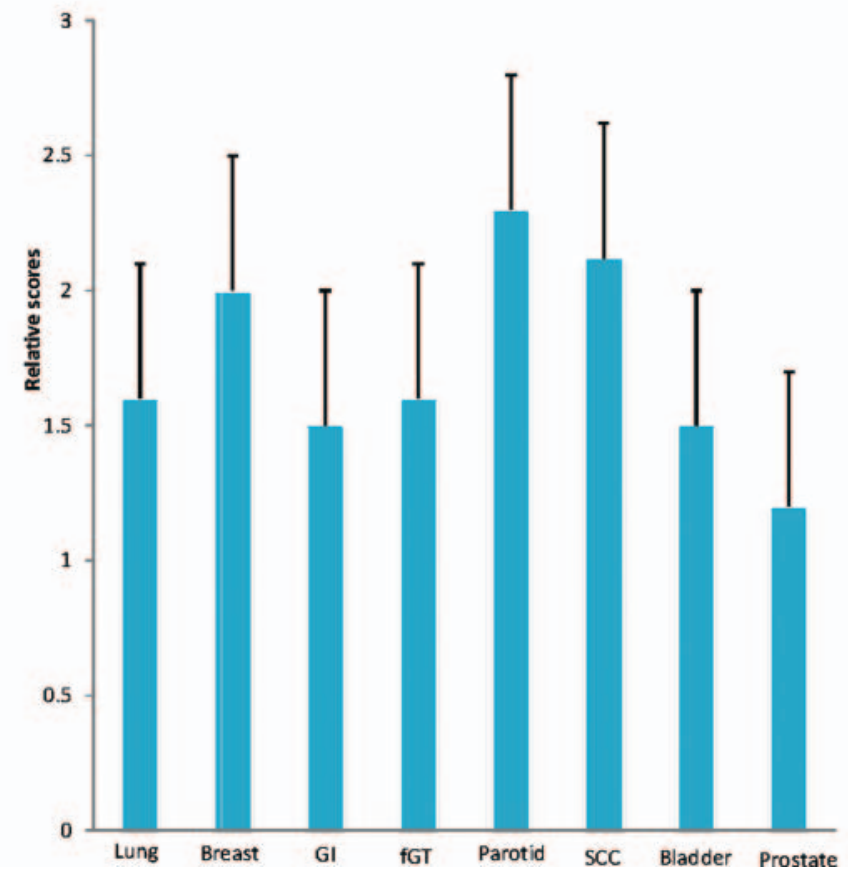

Figure 3 Continued.

Raf-l Antibody (Phospho-Ser ${ }^{259}$ pAb) testing the activated phosphorylated B-Raf. Some of the serial slides were stained in parallel on each patient by different antibodies. As shown in online supplementary figure S6A first two columns, in all 12 clear cell carcinoma of RCC samples, there were no or very weak B-Raf IHC signals in all three used Raf antibodies. In papillary RCC (see online supplementary figure S6B) and chromophobe RCG samples (see online supplementary figure S6C) (15 samples of 24 samples or $62 \%$ of all RCC samples tested), there were higher IHC signals in all three antibodies used for staining in cancer areas specifically, showing higher intensity and extent than clear cell carcinoma (see online supplementary figure S6A). Nuclear signals were found only by B-Raf P-Ser antibody in papillary and chromophobe tumours. Online supplementary table S1 summarises the results. It is intriguing that in those TRIM59 upregulated kidney cancers (RCC), neither total nor phosphorylated BRaf were detected in clear RCC (as a control), but were all highly positive in other two RCCs (papillary and chromophobe) tumours. We could not definitely confirm that TRIM59 was acting along the Ras pathway in all cases where it was detected.

\section{CONCLUSIONS}

This is the first report on a possible 'ubiquitous' tumour marker. Ras mutations are among the most frequent alterations in human cancers that lead to approximately $30 \%$ of all human cancers with expression of constitutively active Ras proteins (for a review see refs. ${ }^{22}$ ${ }^{30}$ ). In this report, we have demonstrated that there are more than mutation issues of the Ras signal pathway in tumorigenesis and progression, since TRIM59 is upregulated as a novel proto-oncogene in a variety of human cancers.

Detection and treatment of cancer at the earliest stage are critical for patient survival. This investigation demonstrated a novel TRIM59 gene as a multiple tumour marker for early diagnosis of tumorigenesis. As a multiple biomarker associated with epithelium origin (very rarely arisen from mesenchymal tissue, see table 2) TRIM59 may be used as an EMT (epithelium-mesenchymal transition) specific cobiomarker. The TRIM59 antibody may also be used for molecular-targeted imaging as a new diagnostic marker, for example, in the context of targeted microbubble ultrasound destruction technology, ${ }^{23}$ before the protocol of a serum TRIM59 test can be established.

\section{Author affiliations}

${ }^{1}$ Department of Surgery, Lawson Health Research Institute, Western University, London, Ontario, Canada

${ }^{2}$ Department of Surgery, University of British Columbia, Vancovour, British Columbia, Canada

${ }^{3}$ Department of Physiology, Robarts Research Institute, Western University, London, Ontario, Canada

${ }^{4}$ Department of Laboratory Medicine and Pathbiology, Sunnybrook Research Institute, University of Toronto, Toronto, Ontario, Canada

${ }^{5}$ London Regional Cancer Program, Western University, London, Ontario, Canada ${ }^{6}$ Department of Pathology, Western University, London, Ontario, Canada

Acknowledgements This work was supported by grants from the Ontario Institute of Cancer Research (07NOV-52), the Canadian Institute of Health 
Research (MOP-77684), NIH-NCI (2 U01 CA084296-06) and the Terry Fox Foundation.

Contributor All authors have contributed significantly to the conception and design of the manuscript, interpretation of data, drafting the article and revisions for important intellectual content and final approval of the version to be published. MM (pathologist), VK and FV worked on data acquisition, analysis and interpretation of human tumour samples. JWX conceived and designed the study. JWX is the guarantor of the study.

Competing interests None.

Provenance and peer review Not commissioned; externally peer reviewed.

Data Sharing Statement Extra data are available by emailing correspondent author JWX.

\section{REFERENCES}

1. Ozato $\mathrm{K}$, Shin DM, Chang TH, et al. TRIM family proteins and their emerging roles in innate immunity. Nat Rev Immunol 2008;8:849-50.

2. James LC, Keeble AH, Khan Z, et al. Structural basis for PRYSPRY-mediated tripartite motif (TRIM) protein function. Proc Natl Acad Sci USA 2007;104:6200-5.

3. Keeble $A H$, Khan Z, Forster A, et al. TRIM21 is an IgG receptor that is structurally, thermodynamically, and kinetically conserved. Proc Natl Acad Sci USA 2008;105:6045-50.

4. Si Z, Vandegraaff N, O'hUigin CS, et al. Evolution of a cytoplasmic tripartite motif (TRIM) protein in cows that restricts retroviral infection. Proc Natl Acad Sci USA 2006;103:7454-9.

5. Ellis J, Hotta A, Rastegar M. Retrovirus silencing by an epigenetic TRIM. Cell 2007:131:13-14.

6. Gack MU, Shin YC, Joo CH, et al. TRIM25 RING-finger E3 ubiquitin ligase is essential for RIG-I-mediated antiviral activity. Nature 2007;446:916-20.

7. Wolf D, Goff SP. TRIM28 mediates primer binding site-targeted silencing of murine leukemia virus in embryonic cells. Cell 2007;131:46-57.

8. Yap MW, Nisole S, Lynch C, et al. Trim5alpha protein restricts both HIV-1 and murine leukemia virus. Proc Natl Acad Sci USA 2004;101:10786-91.

9. Short KM, Cox TC. Subclassification of the RBCC/TRIM superfamily reveals a novel motif necessary for microtubule binding. J Biol Chem 2006;281:8970-80.

10. Lerner M, Corcoran M, Cepeda D, et al. The RBCC gene RFP2 (Leu5) encodes a novel transmembrane E3 ubiquitin ligase involved in ERAD. Mol Biol Cell 2007;18:1670-82.

11. Balastik M, Ferraguti F, Pires-da SA, et al. Deficiency in ubiquitin ligase TRIM2 causes accumulation of neurofilament light chain and neurodegeneration. Proc Natl Acad Sci USA 2008;105:12016-21.

12. Schwamborn JC, Berezikov E, Knoblich JA. The TRIM-NHL protein TRIM32 activates microRNAs and prevents self-renewal in mouse neural progenitors. Cell 2009;136:913-25.

13. Wang L, Heidt DG, Lee CJ, et al. Oncogenic function of ATDC in pancreatic cancer through Wnt pathway activation and $\beta$-catenin stabilization. Cancer Cell 2009;15:207-19.
14. Loedige I, Filipowicz W. TRIM-NHL proteins take on miRNA regulation. Cell 2009;136:818-20.

15. Deshaies RJ, Joazeiro CAP. RING domain E3 ubiquitin ligases. Ann Rev Biochem 2009;78:399-434.

16. Hammell CM, Lubin I, Boag PR, et al. nhl-2 modulates microRNA activity in Caenorhabditis elegans. Cell 2009;136:926-38.

17. Valiyeva F, Jiang F, Elmaadawi A, et al. Proto-oncogenic activity of a novel TRIM59 gene characterized in mouse cancer models bridging Ras and SV40 Tag oncogene signal pathways. Mol Cancer Ther 2011;10:1229-40

18. Gabril MY, Onita T, Ji PG, et al. Prostate targeting:PSP94 gene promoter/enhancer region directed prostate tissue-specific expression in a transgenic mouse prostate cancer model. Gene Ther 2002;9:1589-99.

19. Duan WM, Gabril MY, Moussa M, et al. Knock-in of SV40 Tag oncogene in a mouse adenocarcinoma of the prostate (KIMAP) model demonstrates advantageous features over the transgenic model. Oncogene 2005;24:1510-24.

20. Gabril MY, Duan WM, Wu GJ, et al. A novel knock-in prostate cancer model demonstrates biology similar to that of human prostate cancer and suitable for preclinical studies. Mol Ther 2005;11:348-62.

21. Van Huizen I, Wu GJ, Moussa M, et al. Establishment of a serum tumor marker for pre-clinical trials of mouse prostate cancer models. Clin Cancer Res 2005;11:7911-19.

22. Coleman ML, Marshall CJ, Olson MF. RAS and RHO GTPases in G1-phase cell-cycle regulation. Nat Rev $\mathrm{Mol} \mathrm{Cell} \mathrm{Biol}$ 2004:5:355-66.

23. Xuan JW, Bygrave M, Valiyeva F, et al. Molecular targeted enhanced ultrasound imaging of Flk1 reveals diagnosis and prognosis potential in genetically engineered mouse prostate cancer model. Mol Imaging 2009;8:209-20.

24. Fedor HL, De Marzo AM. Practical methods for tissue microarray construction. In: Gloria HSu, ed. Pancreatic cancer: methods and protocols. Totowa, NJ: Human Press, 2005;89-101.

25. Mills SE. Diagnostic surgery pathology. 5th edn. Philadelphia: Lippincott Williams \& Wilkins, 2010.

26. Wirtzfeld LA, Wu GJ, Bygrave M, et al. Three-dimensional ultrasound micro-imaging for preclinical studies using a transgenic prostate cancer mouse model. Cancer Res 2005;65:6337-45.

27. Xuan JW, Bygrave M, Jiang HY, et al. Functional neo-angiogenesis imaging of genetically engineered mouse prostate cancer using three-dimensional power Doppler ultrasound. Cancer Res 2007;67:2830-9.

28. Imasato $\mathrm{Y}$, Xuan JW, Sakai H, et al. PSP94 expression after androgen deprivation therapy: a comparative study with prostate specific antigen in benign prostate and prostate cancer. J Urol 2000;164:1819-24.

29. Onita T, Ji PG, Xuan JW, et al. Hypoxia-induced, perinecrotic expression of endothelial Per-ARNT-Sim domain protein-1/ hypoxia-inducible factor-2alpha correlates with tumor progression, vascularization, and focal macrophage infiltration in bladder cancer. Clin Cancer Res 2002;8:471-80.

30. Johnson L, Mercer K, Greenbaum D., et al Somatic activation of the $\mathrm{K}$-ras oncogene causes early onset lung cancer in mice. Nature 2001;410:1111-16. 